\title{
STRUCTURE OF SAGITTARIUS A* AT 86 GHz USING VLBI CLOSURE QUANTITIES
}

\author{
S. S. Doeleman, ${ }^{1}$ Z.-Q. Shen, ${ }^{2,3}$ A. E. E. Rogers, ${ }^{1}$ G. C. Bower, ${ }^{4}$ M. C. H. Wright, ${ }^{5}$ J. H. ZhaO,${ }^{6}$ D. C. BACKer, ${ }^{5}$ \\ J. W. Crowley, ${ }^{1}$ R. W. Freund, ${ }^{7}$ P. T. P. Ho, ${ }^{6}$ K. Y. Lo, ${ }^{3}$ AND D. P. WoOdY ${ }^{8}$ \\ Received 2000 September 21 ; accepted 2001 January 24
}

\begin{abstract}
At radio wavelengths, images of the compact radio source Sagittarius $A^{*}\left(\operatorname{Sgr} A^{*}\right)$ in the Galactic center are scatter broadened with a $\lambda^{2}$ dependence due to an intervening ionized medium. We present VLBI observations of Sgr A* at $86 \mathrm{GHz}$ using a six station array, including the VLBA antennas at Pie Town, Fort Davis, and Los Alamos, the $12 \mathrm{~m}$ antenna at Kitt Peak, and the millimeter arrays at Hat Creek and Owens Valley. To avoid systematic errors due to imperfect antenna calibration, the data were modeled using interferometric closure information. The data are best modeled by a circular Gaussian brightness distribution of FWHM $0.18 \pm 0.02$ mas. The data are also shown to be consistent with an elliptical model corresponding to the scattering of a point source. The source structure in the northsouth direction, which is less well determined than in the east-west direction because of the limited north-south $u-v$ coverage of the array, is constrained to be less than 0.27 mas by these measurements. These results are consistent with extrapolations of intrinsic structure estimates obtained with VLBI at a $7 \mathrm{~mm}$ wavelength, assuming the intrinsic size of Sgr $\mathrm{A}^{*}$ has a greater dependence than $\lambda^{0.9}$ with wavelength.
\end{abstract}

Key words: Galaxy: center — galaxies: individual (Sagittarius) — techniques: interferometric

\section{INTRODUCTION}

Sagittarius $\mathrm{A}^{*}\left(\operatorname{Sgr} \mathrm{A}^{*}\right)$ is the compact radio source in the center of the Galaxy and is believed to be powered, as are extra-Galactic active galactic nuclei (AGNs), via accretion onto a massive black hole. The case for this scenario was solidified recently with the stellar proper motion studies of Eckart \& Genzel (1997) and Ghez et al. (1998), which show velocity dispersions of $\sim 1400 \mathrm{~km}$ $\mathrm{s}^{-1}$ near the Galactic center, implying a $2.6 \times 10^{6} M_{\odot}$ central mass within a $10^{-6} \mathrm{pc}^{3}$ volume. Upper limits on the proper motion of Sgr A* itself (Backer \& Sramek 1999; Reid et al. 1999) constrain its mass to be greater than $\sim 2 \times 10^{4} \quad M_{\odot}$. These results would seem to rule out stellar-type phenomena as models for the radiative output from Sgr $\mathrm{A}^{*}$, which spans radio to X-ray wavelengths. Estimates of the luminosity of Sgr A* (Zylka, Mezger, \& Lesch 1992) are only a small fraction $\left(\sim 10^{-5}\right)$ of the Eddington limit for a $2.6 \times 10^{6} M_{\odot}$ black hole (Quataert, Narayan, \& Reid 1999). Physical models that reproduce the observed $\operatorname{Sgr} \mathrm{A}^{*}$ emission spectrum in the context of mass estimates above include scaled AGN models (Falcke \& Biermann 1999), advection-dominated spherical accretion flows (ADAFs; Narayan, Yi, \& Maha-

\footnotetext{
${ }^{1}$ MIT Haystack Observatory, Off Route 40, Westford, MA 01886.

2 National Astronomical Observatory, Mitaka, Tokyo 181-8588, Japan.

${ }^{3}$ Academia Sinica Institute of Astronomy and Astrophysics (ASIAA), PO Box 1-87, Nankang, Taipei 11529, Taiwan.

${ }^{4}$ National Radio Astronomy Observatory (NRAO), P.O. Box 0, 1003 Lopezville Road, Socorro, NM 87801.

5 Department of Astronomy, University of California, Berkeley, 601 Campbell Hall, Berkeley, CA 94720.

${ }^{6}$ Harvard-Smithsonian Center for Astrophysics, 60 Garden Street, Cambridge, MA 02138.

${ }^{7}$ NRAO, 949 North Cherry Avenue, Tucson, AZ 85721.

${ }^{8}$ California Institute of Technology, Owens Valley Radio Observatory, Big Pine, CA 91125.
}

devan 1995), and cyclosynchrotron emission from spherical accretion (Melia 1992).

Direct measurement of the intrinsic size of Sgr $\mathrm{A}^{*}$ is of great importance in the discrimination of different emission mechanisms thought to operate in this source. In the radio bands, such measurements are complicated by scattering through the inhomogeneous ionized interstellar medium, which broadens the images. Past VLBI observations, ranging in wavelength from $6 \mathrm{~cm}$ to $1.3 \mathrm{~cm}$, identify an elliptical source with axial ratio of 0.53 , oriented east-west, whose dimensions vary with $\lambda^{2}$, as expected for scattering of an unresolved source by electron density irregularities (Lo et al. 1985; Jauncy et al. 1989; Marcaide et al. 1992; Alberdi et al. 1993). Similar elliptical scattering disks are observed for $\mathrm{OH}$ masers located within $25^{\prime}$ of Sgr A* (Frail et al. 1994). Such noncircular scattering implies an anisotropy in the underlying density inhomogeneities that may arise because of the ordered magnetic fields in the region.

Using $7 \mathrm{~mm}$ wavelength VLBI, Lo et al. (1998) reported a departure from this axial ratio of the scattering ellipse, a result consistent with independent measurements by Bower \& Backer (1998). The apparent departure from the scattering, if confirmed, would be strong evidence for intrinsic structure in $\mathrm{Sgr} \mathrm{A}^{*}$. A possible elongation of $\mathrm{Sgr} \mathrm{A*}$ at $7 \mathrm{~mm}$ was first reported by Krichbaum et al. (1993), who modeled Sgr A* with two VLBI components separated by 3 mas along a P.A. of $-25^{\circ}$. Later $7 \mathrm{~mm}$ VLBI work by Backer et al. (1993) and Bower \& Backer (1998) found no evidence for such structure. Lo et al. (1998) interpret their $7 \mathrm{~mm}$ result as an intrinsic structure, consisting of an elongation of $0.44 \pm 0.09$ mas with a position angle of $-10^{\circ}$. The reported statistical significance of this result is $5 \sigma$, but there are additional systematic errors in the calibration (Bower et al. 1999). VLBI observations of Sgr A* at $3.5 \mathrm{~mm}$ wavelength, which are much less affected by interstellar scattering, can provide important verification of the $7 \mathrm{~mm}$ 
results. Intrinsic structure of Sgr $\mathrm{A}^{*}$, if visible at $7 \mathrm{~mm}$, should be readily observable at shorter wavelengths, as long as its size does not decrease faster than $\lambda^{2}$.

Previous size estimates for Sgr A* from VLBI at $3.5 \mathrm{~mm}$ wavelength have been obtained using arrays of two (Rogers et al. 1994) and three (Krichbaum et al. 1998) antennas. The sparse data sets of Rogers et al. (1994) and Krichbaum et al. (1998) were modeled with circular Gaussian brightness distributions and yielded sizes of $0.15 \pm 0.05$ and $0.19 \pm 0.03$ mas, respectively, consistent with a $\lambda^{2}$ extrapolation of the scattering size at this wavelength. There remain some differences in the literature as to the exact power law that best characterizes the relation between scattering size and wavelength (Backer et al. 1993; Rogers et al. 1994; Krichbaum et al. 1998). Differences in the number and weighting of past size measurements are largely responsible for the variation in fitted relations. In this paper, we will adopt the relations cited by Lo et al. (1998), which yield a size of $1.43 \lambda^{2}$ mas for the major axis and $0.76 \lambda^{2}$ mas for the minor axis, where $\lambda$ is measured in centimeters. These result from nearly simultaneous observations reduced in a uniform manner.

The limited baseline coverage and sensitive dependence on a priori antenna calibrations of previous arrays have not yet allowed a definitive estimate of intrinsic structure at shorter wavelengths, although a detection of Sgr A* at the $1.3 \mathrm{~mm}$ wavelength has been reported (Krichbaum et al. 1998). This paper reports on new VLBI observations made at the $3.5 \mathrm{~mm}$ wavelength with an array of six antennas, which allowed use of closure quantities to model the Sgr A* structure. We explore two different methods of model fitting using the closure-amplitude information. Closure amplitudes and phases provide structural information without the need to calibrate the VLBI array (Readhead et al. 1980).

\section{OBSERVATIONS}

Observations of Sgr $\mathrm{A}^{*} \quad\left(\alpha=17^{\mathrm{h}} 45^{\mathrm{m}} \quad 40.045, \quad \delta=\right.$ $-29^{\circ} 00^{\prime} 27^{\prime \prime} .9$ [J2000.0]; Rogers et al. 1994) at $86 \mathrm{GHz}$ were made by the Coordinated Millimeter VLBI Array ${ }^{9}$ in 1999 April. Fringes were obtained to the Kitt Peak $12 \mathrm{~m}$ antenna $(\mathrm{K})$, the Owens Valley Millimeter Array (O), the BerkeleyIllinos-Maryland Association (BIMA) array at Hat Creek $(\mathrm{H})$, and the NRAO ${ }^{10}$ VLBA sites at Los Alamos (L), Pie Town (P), and Fort Davis (F). The symbols to be used in this paper for each site are listed in parentheses. Sgr A* and continuum calibrators NRAO 530 and OV-236 were observed on both 1999 April 16 and 18, but technical problems at the VLBA sites reduced the array to just three antennas on the first day. The data presented here were observed during the second day in which $\operatorname{Sgr} \mathrm{A}^{*}$ was detected on baselines to all sites. A total bandwidth of $56 \mathrm{MHz}$, consisting of 14 separate $4 \mathrm{MHz}$ channels, was observed for $360 \mathrm{~s}$ duration scans. SiO masers toward VX Sgr were observed at both 43 and $86 \mathrm{GHz}$ for pointing and calibration, respectively. The data were processed on the Mark IIIA correlator at Haystack Observatory with an integration time of $1 \mathrm{~s}$.

\footnotetext{
${ }^{9}$ Support for the Coordinated Millimeter VLBI Array work at the Haystack Observatory is provided under a grant from the NSF to the Northeast Radio Observatory Corporation.

${ }^{10}$ The National Radio Astronomy Observatory is a facility of the NSF operated under cooperative agreement by Associated Universities, Inc.
}

\section{A PRIORI CALIBRATION}

Accurate a priori amplitude calibration of VLBI visibilities relies completely on the successful determination of telescope sensitivity as a function of time and elevation, using previously established gain information. At the low elevations required for Sgr A* observations, millimeterwave dishes, such as the Kitt Peak $12 \mathrm{~m}$, can be accurately characterized, but gains for the VLBA antennas, which are not yet optimized for $86 \mathrm{GHz}$ observations, are difficult to determine. Phasing at the millimeter arrays also adds a degree of gain variation that depends sensitively on timevariable atmospheric conditions. Two independent a priori calibration techniques were applied to the array.

The system equivalent flux density (SEFD) was first determined for each antenna as a function of time, using system temperature (Tsys) measurements and previously established gain-elevation curves. VLBA antennas use internal noise sources to determine Tsys, and these values were corrected for atmospheric opacity effects. The millimeter arrays and Kitt Peak use a vane absorber method to measure Tsys, and no opacity correction is required. Explicit assumptions in this type of array calibration are perfect pointing and stable gain curves at all sites. A faulty calibration source prevented Tsys from being recorded at the VLBA Fort Davis site.

A second calibration method made use of total power spectra obtained by autocorrelating VLBI data on the 86 $\mathrm{GHz} \mathrm{SiO}$ maser source VX Sgr $\left(\alpha=18^{\mathrm{h}} 08^{\mathrm{m}} 04^{\mathrm{s}} .04, \delta=\right.$ $-22^{\circ} 13^{\prime} 26^{\prime \prime} .90$ [J2000.0]; Wright et al. 1990) to generate 112 channel spectra in a $4 \mathrm{MHz}$ bandpass. A template to the maser line structure from the Kitt Peak autocorrelations was calibrated assuming a flat gain curve and a sensitivity of $0.025 \mathrm{~K} \mathrm{Jy}^{-1}$. This template spectrum was then fitted to a total power spectra extracted from all VX Sgr observations at all array sites to determine the SEFD of each antenna as a function of time. This method of calibration assumes perfect pointing only for the template spectrum; pointing errors on all other VX Sgr observations will be corrected relative to the template scan. At BIMA, a phase calibration tone leaking into the bandpass prevented calibration using the spectral template technique.

Figure 1 shows the results of these two calibrations for all sites. At four sites (Kitt Peak, Owens Valley Radio Observatory [OVRO], Pie Town, and Los Alamos), both the spectral template (filled circles) and gain curve (solid line) calibrations were used. The Kitt Peak and OVRO sites show generally good agreement between the two methods, although it appears that the gain curve at Kitt Peak may have more elevation dependence than originally assumed. The gain curve derived SEFD at Kitt Peak rises more slowly at lower elevations than the VX Sgr derived values. For the two VLBA sites, correspondence between the two calibrations is distinctly inferior. In the elevation range $20^{\circ}-$ $30^{\circ}$, the gain curve calibration is consistently below the VX Sgr SEFD values by as much as a factor of 4 , in the case of Los Alamos, and by a factor of 2.5 for Pie Town. The difference between the calibration schemes is probably due to pointing errors at the VLBA sites, which are given, along with their effects on VLBI amplitudes, in Table 1. The loss factors given are $1 \sigma$ values, so the observed scatter in the spectral template results is entirely consistent with pointing errors as well. At $86 \mathrm{GHz}$, the VLBA pointing is affected by known systematic pointing offsets at the $20^{\prime \prime}$ level due to a 

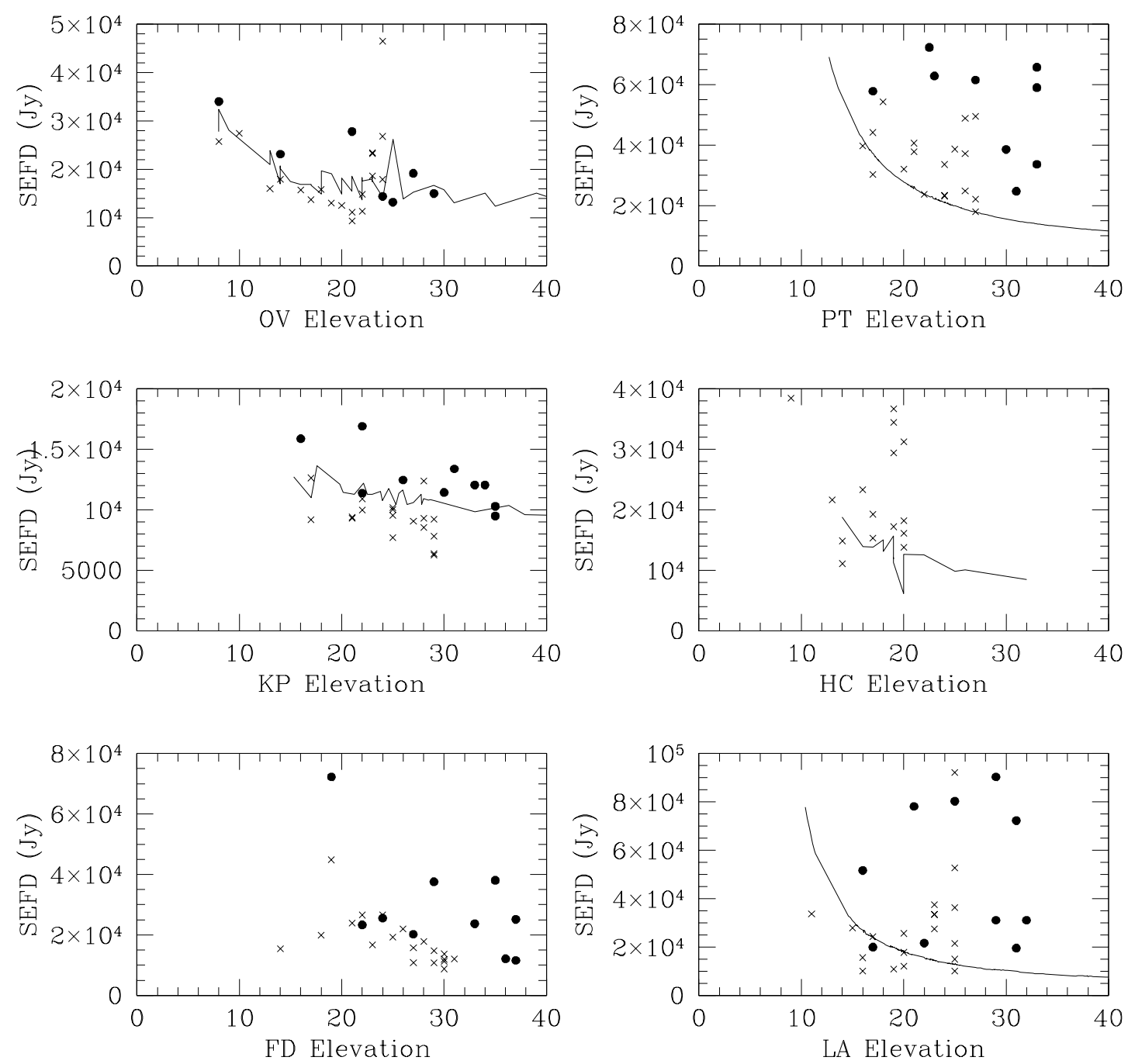

FIG. 1.-Comparison of three calibration methods for the VLBI array. Filled circles mark the SEFD, measured using auto spectra on the line calibrator VX Sgr. This method reflects the effects of pointing errors. The second calibration, marked by crosses, is the result of solving for station gains in the model-fitting process described in the text. Similar degrees of scatter in the gains found by the two methods suggest they are both tracking similar variations. Pointing errors tabulated in Table 1 probably account for most of the observed gain variation. The third calibration used published gain curves and measured system temperatures to calculate the SEFD and is shown as a solid line.

ripple in the azimuth track (V. Dhawan 2000, private communication). Both a priori calibration methods, thus, do not compensate for pointing errors on $\mathrm{Sgr} \mathrm{A}^{*}$ observations at the VLBA antennas. In order to avoid these

TABLE 1

Signal Loss Due to Pointing ERrors at VLBA Sites

\begin{tabular}{ccccc}
\hline \hline $\begin{array}{c}\text { Antenna } \\
(1)\end{array}$ & $\begin{array}{c}\text { rms Pointing } \\
(\operatorname{arcsec}) \\
(2)\end{array}$ & $\begin{array}{c}\text { Loss } \\
\text { Factor 1 } \\
(3)\end{array}$ & $\begin{array}{c}\text { Loss } \\
\text { Factor 2 } \\
(4)\end{array}$ & $\begin{array}{c}\text { Loss } \\
\text { Factor 3 } \\
(5)\end{array}$ \\
\hline VLBA-PT $\ldots . .$. & 17 & 0.33 & 0.76 & 0.58 \\
VLBA-LA ...... & 14 & 0.47 & 0.83 & 0.69 \\
VLBA-FD ....... & 13 & 0.64 & 0.90 & 0.80 \\
\hline
\end{tabular}

Notes.-Col. (1): Site; col. (2): rms of the difference between consecutive pointings performed every 20 minutes during the observations; col. (3): loss of sensitivity due to the pointing errors: $\exp \left[-(\ln 2) \mathrm{rms}^{2} /\right.$ HWHM $^{2}$, where HWHM is the half width at half maximum of the antenna beam; col. (4): loss assuming the telescopes are properly pointed at the beginning of each scan but drift to the pointing error values during a 20 minute scan; col. (5): loss given by a mixture of the two cases. difficulties in calibration, which are likely not tractable at $3.5 \mathrm{~mm}$, we chose to use only closure amplitudes in our analysis that are not susceptible to calibration errors and are determined on observations of Sgr A* itself.

\section{DATA ANALYSIS}

The Sgr $A^{*}$ data were analyzed using the following procedure:

1. Fringes were obtained at all sites on the calibration sources to determine the calibration phases that allow coherent bandwidth synthesis of the baseband channels. In addition, the calibration sources were used to estimate the clock delays and rates for each station.

2. A fringe search was conducted on Sgr A* to refine the delays and rates using the Haystack Observatory's postprocessing system software. This step used interpolations of delay and rate from adjacent calibrator scans, as well as strong Sgr A* detections on sensitive baselines, to constrain searches on weaker baselines. This method works even when there are very weak fringes on some baselines, as long 
as there are sufficiently strong fringes on enough baselines to reduce the search space (see, e.g., Alef \& Porcas 1986). The data were also imported into the NRAO AIPS software package in which independent fringe searches resulted in consistent delay and rate results.

3. The noise bias corrected incoherent average of $10 \mathrm{~s}$ data segments was computed for each baseline using the refined clock delays from the constrained fringe search. These data segments are short enough to avoid significant signal loss due to atmospheric path fluctuations. The incoherent average $\langle a\rangle$ (Rogers, Doeleman, \& Moran 1995) is

$$
\langle a\rangle=\left[1 / M \sum_{s=0}^{M-1}\left(\left|a_{s}\right|^{2}-2 \sigma_{c}^{2}\right)\right]^{1 / 2},
$$

where $a_{s}$ is the complex correlation from the coherent integration of each segment, and $\sigma_{c}$ is the noise bias

$$
\sigma_{c}=\frac{1}{L}(2 B T)^{-1 / 2},
$$

where $B=56 \mathrm{MHz}$; the coherent integration, $T$, is $10 \mathrm{~s}$; the loss factor, $L$, is $\simeq 0.55$ for a digital MK3 1-bit correlation, including fringe rotator loss; and $M$ is the number of segments.

4. Closure phases, the sum of interferometric phases around a closed loop of three baselines (Jennison 1958; Rogers et al. 1974), were estimated for each 360 s VLBI recording scan. The complex valued bispectrum or triple product (Rogers et al. 1995), whose argument is the closure phase, was calculated for each $10 \mathrm{~s}$ data segment and averaged to a full scan length. This avoided any coherence losses due to phase fluctuations over the length of an entire $360 \mathrm{~s}$ VLBI observation. Deviations of the closure phase from zero on any triangle would indicate an asymmetric component in the structure of $\mathrm{Sgr} \mathrm{A}^{*}$, zero closure phases are the signature of a brightness distribution symmetric about the origin. For a brightness distribution dominated by a central compact feature, closure phases are not expected to change significantly on a $360 \mathrm{~s}$ timescale. Closure-phase plots from Bower \& Backer (1998) show that even for a two-component model of $\mathrm{Sgr} \mathrm{A*}$ at $7 \mathrm{~mm}$, closure phases vary by $<5^{\circ}$ in a 6 minute period. In the low signal-to-noise $(\mathrm{S} / \mathrm{N})$ regime, the bispectral $\mathrm{S} / \mathrm{N}$ is proportional to the product of the $\mathrm{S} / \mathrm{Ns}$ of each contributing baseline, and it can be shown (Rogers et al. 1995) that this $\mathrm{S} / \mathrm{N}$ is greater than that of the closure phase alone. We employ a bispectral S/N cutoff, which ensures an $\mathrm{S} / \mathrm{N}$ in each $10 \mathrm{~s}$ segment on each baseline of approximately 1.6. This corresponds to a $15^{\circ}$ standard deviation in the closure phase.

The measured closure phases are consistent with zero values. Several representative closure-phase triangles, whose high $\mathrm{S} / \mathrm{N}$ enabled us to track their values over multiple scans are listed here with their measured standard deviations: $\mathrm{FKP}=24^{\circ} \pm 12^{\circ}, \mathrm{FKL}=10^{\circ} \pm 23^{\circ}, \mathrm{KOL}=$ $11^{\circ} \pm 22^{\circ}, \mathrm{FKH}=3^{\circ} \pm 20^{\circ}$, and FKO $=-6.3^{\circ} \pm 27^{\circ}$. Subsequent modeling was restricted to symmetric brightness distributions.

5. A circular or elliptical Gaussian is fitted to the amplitudes, allowing separate station-based gains for each $360 \mathrm{~s}$ scan. This is similar to the commonly used self-calibration technique for imaging interferometric data, except that selfcalibration cannot search all possible brightness distributions. Instead, self-calibration converges iteratively on a consistent set of complex antenna gains and an image by using a deconvolution method, such as CLEAN. The resulting image is not unique (Schwarz 1978) because of the iterative nature of the imaging process. Alternatively, the method described below performs an exhaustive search over all models in a restricted parameter space and guarantees that the result is the most likely.

The best-fit model is found by minimizing $\chi^{2}$. For a weighted least-squares fit,

$$
\chi^{2}=\sum_{\mathrm{ij}} w_{\mathrm{ij}}(t)\left[a_{\mathrm{ij}}(t)-m_{\mathrm{ij}} e^{g_{i}(t)} e^{g_{j}(t)}\right]^{2}
$$

where $m_{\mathrm{ij}}(t)$ is the model at time $t$ for baseline $\mathrm{ij}, a_{\mathrm{ij}}(t)$ is the observed incoherent average of data segments, $e^{g_{i(t)}}$ is the gain for station $i$ for each scan at time $t$, and $w_{\mathrm{ij}}(t)=1 / \sigma_{\mathrm{ij}}^{2}(t)$, where $\sigma_{\mathrm{ij}}^{2}(t)$ is the estimated variance in $a_{\mathrm{ij}}(t)$.

The standard deviation in each noise bias corrected amplitude estimate in correlation units is

$$
\sigma=\sigma_{c} M^{-1 / 2}\left[\left(s^{2}+1\right) / s^{2}\right]^{1 / 2},
$$

where $s$ is the coherent $\mathrm{S} / \mathrm{N}$ for each segment. Monte Carlo simulations show this approximation to hold for a coherent $\mathrm{S} / \mathrm{N}=a / \sigma_{c} \geq 0.5$. For example, a correlation of $3 \times 10^{-5}$ has a $\mathrm{S} / \mathrm{N}=0.5$, with $10 \mathrm{~s}$ segments, $M=36$, and $\sigma \simeq 1.5 \times 10^{-5}$. Baselines with correlations below $3 \times 10^{-5}$ were not used in the model fit.

The summation is carried over all available baselines and scans. For each scan and each model, the station gains are estimated by the weighted least-squares (Bevington \& Robinson 1992, see also Scharf 1991 for matrix notation) solution of

$$
y=A x+n,
$$

given by

$$
\hat{x}=\left(A^{T} w A\right)^{-1} A^{T} w y,
$$

where $x=\left[g_{0}, \ldots, g_{L-1}\right]^{T}$ is the gain vector for $L$ sites that participate in the scan, $w$ is the diagonal weight matrix, $y$ is the data vector, $A$ is the steering (or curvature) matrix, $n$ is the noise or error vector, $T$ is the transpose operator, $\hat{x}$ is the weighted least-squares estimate of $x$, and the superscript " -1 " refers to the inverse operator.

The $n$th element of the data vector is $y_{n}=\ln \left(a_{n} / m_{n}\right)$, where $n$ is the baseline number. The design matrix elements are $A_{\mathrm{nk}}=1$ when $k=i$ or $k=j$, and $A_{\mathrm{nk}}=0$ otherwise, where $k$ is the station number. The use of logarithms linearizes the estimation of the gains. The search for a minimum $\chi^{2}$ using equation (3) requires separate gain solutions for each model tested, since the gain solutions depend on the model. A one-dimensional Monte Carlo search is made for the circular Gaussian with minimum $\chi^{2}$, while an elliptical Gaussian requires a three-dimensional search through parameter space. Pearson (1995) discusses the least-squares fitting of Gaussian models to VLBI data and the estimation of errors. Under the assumption of a Gaussian distribution of errors, the weighted least-squares approach, which simultaneously solves for the model and the station gains, is equivalent to the maximum likelihood solution. Using this method, we obtained the best-fit circular and elliptical Gaussian models shown in Table 2 (models A and B).

The resulting least-squared station gains are directly compared with the a priori results in Figure 1, represented by crosses. In most cases, the modeled station SEFD values are less than spectral template values at comparable elevations. This may be due to the proportionality of the 
TABLE 2

DesCription AND Fit OF MODELS FOR SGR A*

\begin{tabular}{|c|c|c|c|c|c|c|c|c|}
\hline $\begin{array}{l}\text { Model } \\
\text { (1) }\end{array}$ & $\begin{array}{c}\theta_{\mathrm{maj}} \\
(\mathrm{mas}) \\
(2)\end{array}$ & $\begin{array}{c}\theta_{\min } \\
(\mathrm{mas}) \\
(3)\end{array}$ & $\begin{array}{l}\text { P.A. } \\
\text { (deg) } \\
\text { (4) }\end{array}$ & $\begin{array}{l}\chi^{2} \\
(5)\end{array}$ & $\begin{array}{l}\chi_{v}^{2} \\
(6)\end{array}$ & $\begin{array}{l}\Delta \chi^{2} \\
(7)\end{array}$ & $\begin{array}{c}\sigma \\
(8)\end{array}$ & $\begin{array}{c}\text { Comments } \\
\text { (9) }\end{array}$ \\
\hline A ...... & $0.34 \pm 0.14$ & $0.17 \pm 0.02$ & $22 \pm 20$ & 97 & 1.19 & 0 & $\ldots$ & Best-fit elliptical \\
\hline B ...... & $0.18 \pm 0.02$ & $\ldots$ & $\ldots$ & 102 & 1.25 & 5 & 1.5 & Best-fit circular \\
\hline $\mathrm{C} \ldots \ldots$ & $0 . \overline{175}$ & 0.092 & 80 & 104 & 1.28 & 7 & 2 & Scattering alone \\
\hline $\mathrm{D} \ldots \ldots$ & 0.263 & 0.175 & -10 & 111 & 1.36 & 14 & 3 & Lo et al. model scaled \\
\hline E ....... & 0.44 & 0.175 & -10 & 247 & 3.03 & 150 & 10 & Lo et al. model unscaled \\
\hline
\end{tabular}

NotE.-Col. (1): Model name; col. (2): FWHM major axis; col. (3): FWHM minor axis; col. (4): position angle of major axis (for elliptical models); col. (5): $\chi^{2}$ of the model fit; col. (6): reduced $\chi^{2}$ obtained by dividing $\chi^{2}$ by the number of baselines minus the rank of the matrix $A^{T} w A$ (see text); col. (7): $\chi^{2}$ difference from model A; col. (8): equivalent formal standard error with equivalent confidence at the $\Delta \chi^{2}$ for the 3 degrees of freedom in an elliptical Gaussian model; $\operatorname{col}(9)$ : model description.

modeled SEFDs on the total flux density of Sgr A*, which we have assumed to be $1.4 \mathrm{Jy}$ based on BIMA observations taken prior to the VLBI observations (M. Wright 2000, private communication). If the flux of Sgr $\mathrm{A}^{*}$ during our observing epoch was higher, the derived station gains from the modeling would increase.

Figure 2 compares the gain-corrected visibility amplitudes with a model for the best-fit circular Gaussian. The same data are recast as a plot of correlated flux density as a function of baseline length in Figure 3. The fit to an elliptical Gaussian is not well determined in the north-south direction, owing to the reduced $u-v$ coverage in this direction (see Fig. 4). Uncertainties in the models were estimated from the size of the region in parameter space, corresponding to $\Delta \chi^{2} \leq 1$. In Figure 5, we have plotted $\chi^{2}$ as a function of the FWHM of the circular Gaussian model. We explored a range of coherent averaging times from 4 to $60 \mathrm{~s}$ and

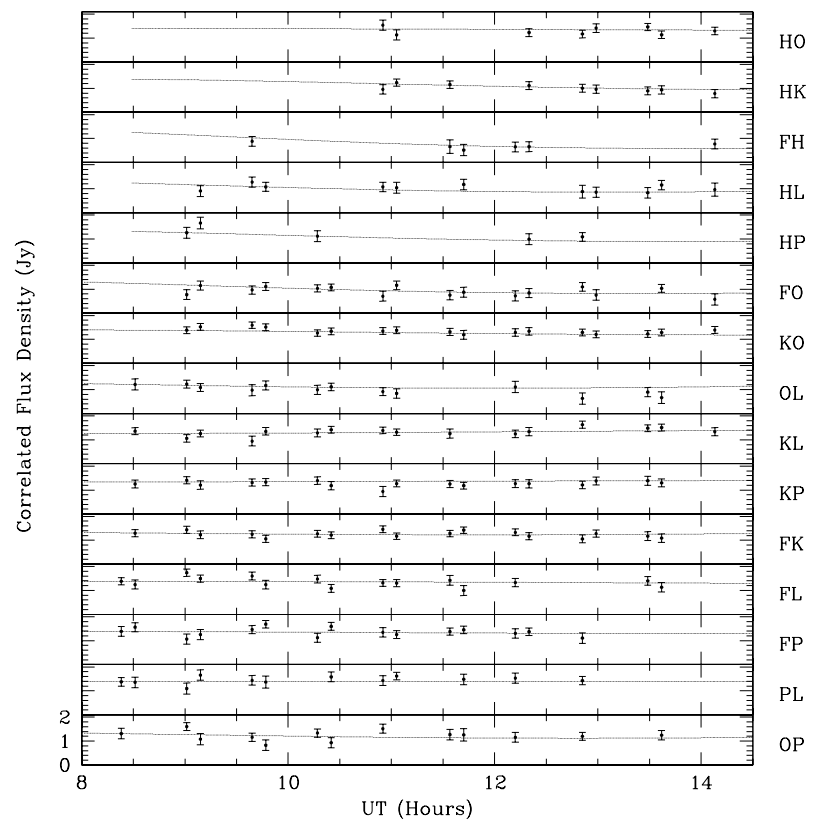

FIG. 2.-Calibrated visibility amplitudes for each baseline between Fort Davis (F), Kitt Peak (K), Owens Valley (O), Hat Creek (H), Pie Town $(\mathrm{P})$, and Los Alamos (L). Calibration of each antenna was determined from a weighted least-squares search through possible circular Gaussian models of brightness distribution. The dotted lines show amplitude curves for the best-fit circular Gaussian model ( $F W H M=0.18$ mas). Error bars are $3 \sigma$, and the flux density scale for all baselines is the same. found very little change in our results from 4 to $20 \mathrm{~s}$. At $60 \mathrm{~s}$, $\chi_{v}^{2}$ was over 1.5 , and the estimated error in the size of the best-fit circular Gaussian more than doubled.

We also modeled the data using closure-amplitude quadrilaterals, which are insensitive to station gains. The closure amplitude is defined as

$$
C_{\mathrm{ijk} 1}=\frac{a_{\mathrm{ij}} a_{\mathrm{k} 1}}{a_{\mathrm{ik}} a_{\mathrm{j} 1}}
$$

a ratio of complex amplitudes around a closed quadrilateral loop of baselines. The number of independent closure amplitudes is equal to the number of baselines between all sites minus the number of sites, so that for six sites, there are nine independent closure amplitudes. Cornwell (1995) discusses the equivalence of self-calibration and modeling of closure quantities in determining source structure. However, using the $\chi^{2}$ from fitting the closure quadrilaterals to estimate the errors in source parameters is not straight-

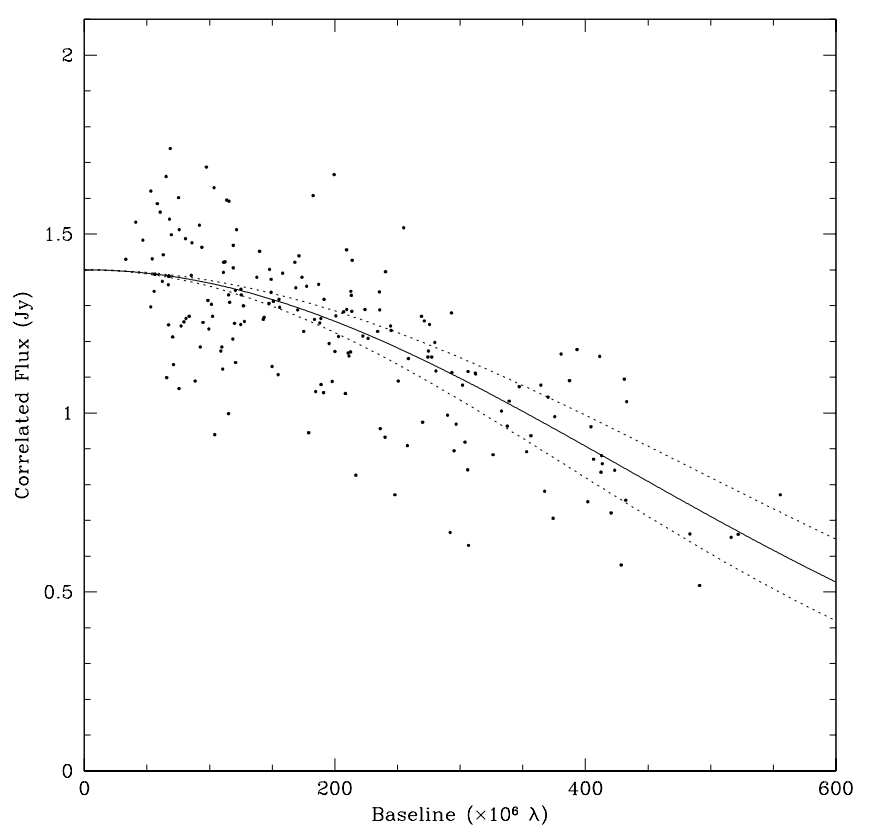

FIG. 3.-Calibrated visibility amplitudes as in Fig. 2, plotted as a function of baseline length. The solid line shows the best-fit circular Gaussian model, and the dotted lines show circular Gaussians that are $\pm 1 \sigma$ in size. Error bars are identical to those shown in Fig. 2 and are left off here for clarity. The scatter in the data is reflective of the errors. 


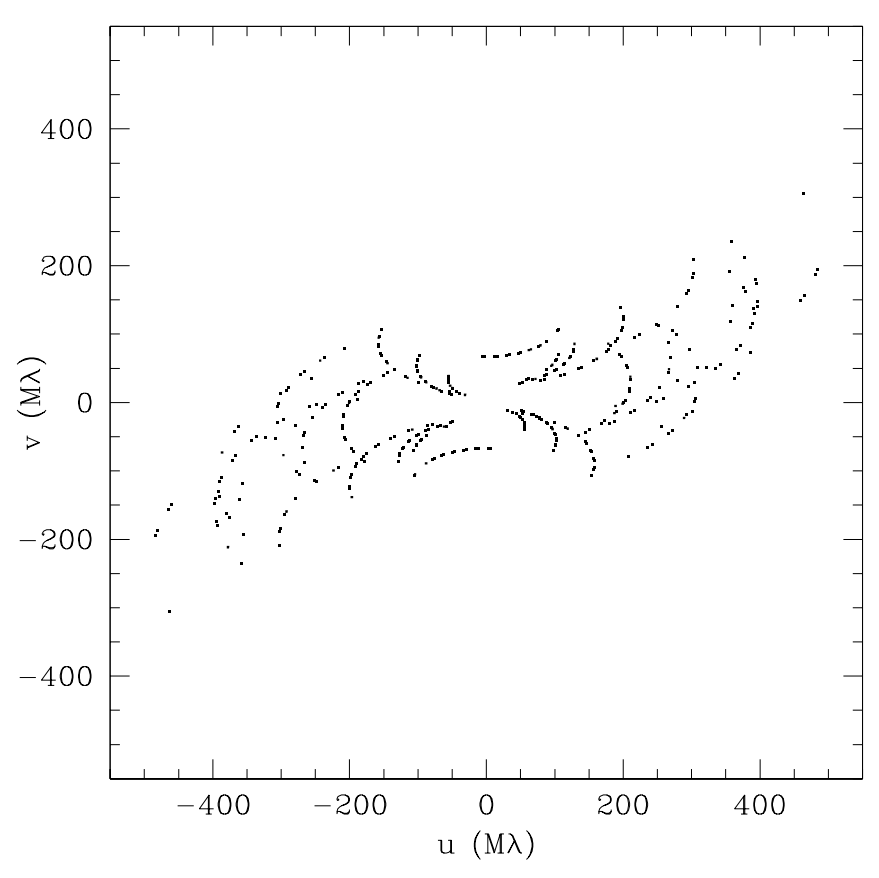

FIG. 4. - $u-v$ coverage corresponding to all observations of Sgr A*

forward, since the quadrilaterals are not all independent. Furthermore, in the low $\mathrm{S} / \mathrm{N}$ case, the formal error for each closure amplitude is difficult to determine (see, e.g., Trotter, Moran, \& Rodríguez 1998, eqs. [4] and [5]).

Based upon VLBA measurements between the $6 \mathrm{~cm}$ and $7 \mathrm{~mm}$ wavelengths, Lo et al. (1998) have suggested that the $7 \mathrm{~mm}$ results show deviation of the minor-axis size from the $\lambda^{2}$ dependence, implying an intrinsic size of $0.44 \pm 0.1$ mas along a P.A. $=-10^{\circ}$. Since the scattering angles are smaller at the $3.5 \mathrm{~mm}$ wavelength, it is important to compare the constraints set by the $3.5 \mathrm{~mm}$ data with reasonable extrapolations from the Lo et al. (1998) inferred intrinsic structure. Model C constitutes pure $\lambda^{2}$ interstellar scattering of a point source. Model D is the extrapolated apparent source

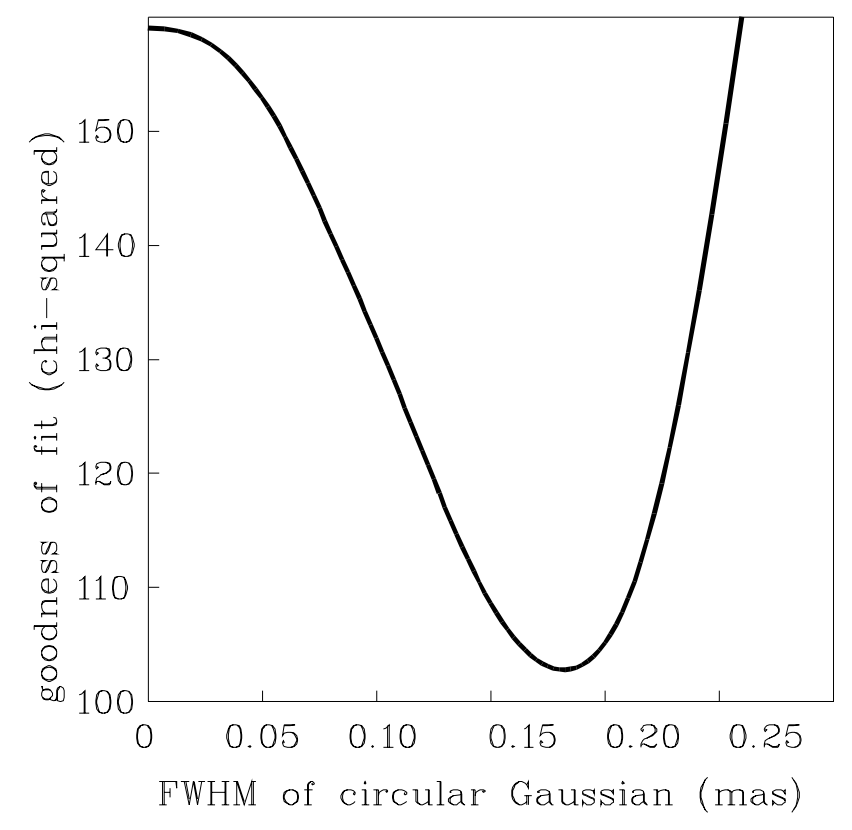

FIG. 5. $-\chi^{2}$ vs. the FWHM for a circular Gaussian model size based on an intrinsic source size that varies as $\lambda^{0.9}$ (Lo et al. 1999). Model E represents the case in which the $7 \mathrm{~mm}$ derived intrinsic structure does not vary with wavelength. Estimates of apparent source size at 3.5 and $1.3 \mathrm{~mm}$ (Rogers et al. 1994; Krichbaum et al. 1998) are consistent with some sort of wavelength-dependent intrinsic structure from 7 to $1.3 \mathrm{~mm}$, but these results depend sensitively on a priori calibration. The exact behavior of the Sgr A* intrinsic structure as a function of frequency is thus largely uncertain, with estimates for a power-law dependence $\left(\lambda^{\alpha}\right)$ ranging from $\alpha=0.7$ to 1.9 . Table 2 contains characteristics and fit results for models C, D, and E. For all elliptical models, the reported P.A. reflects the major-axis orientation.

\section{DISCUSSION}

The $3.5 \mathrm{~mm}$ observations are consistent within $2 \sigma$ of the elliptical scattering model of a point source. They are also consistent at the $3 \sigma$ level, with a model combining the elliptical scattering disk with an intrinsic structure extrapolated from the Lo et al. (1998) $7 \mathrm{~mm}$ estimates, assuming a $\lambda^{0.9}$ dependence. Increasing the spectral index of this power law and, consequently, decreasing the intrinsic size improves agreement with the $3.5 \mathrm{~mm}$ data, as the observed size approaches the scattering size. To further illustrate the level of agreement of the $3.5 \mathrm{~mm}$ data with all models, we show in Figure 6 a closure amplitude for the Fort Davis, Kitt Peak, OVRO, Hat Creek quadrangle $\left(C_{\mathrm{FKон}}\right)$, along with the same closure-amplitude values expected for models A-E. Model E, shown as the solid line in the bottom panel of Figure 6, provides the poorest fit to the data. The dotted lines show the effects on model $\mathrm{E}$ if the north-south intrinsic structure from Lo et al. (1998) is reduced or increased by their cited $1 \sigma$ errors. Other quadrilaterals also show similar inconsistencies with model $\mathrm{E}$.

It has been customary in VLBI studies of $\mathrm{Sgr} \mathrm{A}^{*}$ to model the brightness distribution as an elliptical Gaussian, given the scattering morphology seen at longer wavelengths. At shorter wavelengths $(\lambda \leq 7 \mathrm{~mm})$, however, it is not clear that the data warrant a model more complex than a circular Gaussian. Model A in Table 2 has the lowest $\chi^{2}$ of any model tested, but it also contains two extra model parameters relative to model $\mathrm{B}$. The $F_{\chi}$ test (Bevington \&
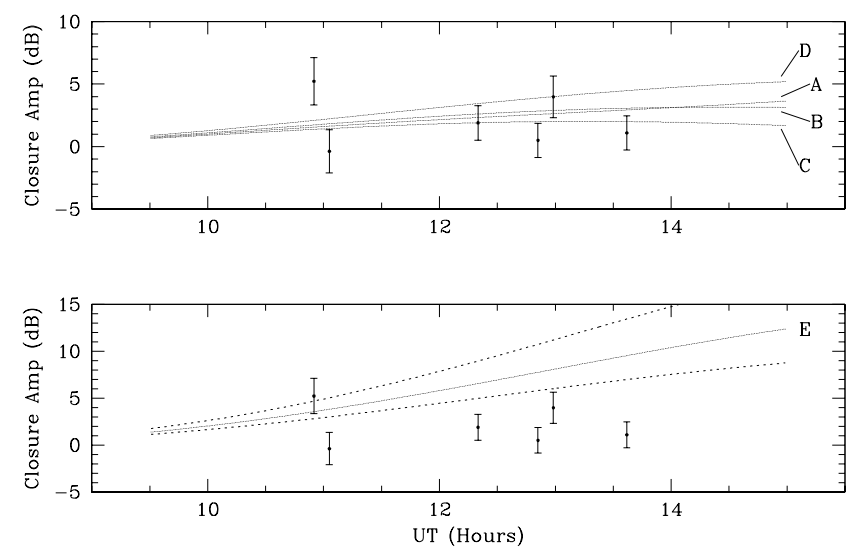

Fig. 6.-Measured closure amplitudes for the Fort Davis, Kitt Peak, OVRO, and Hat Creek quadrilateral, along with closure-amplitude curves for models A-E. The top panel compares the closure amplitudes for models A-D. The bottom panel shows model $\mathrm{E}$ and two variants: model $\mathrm{E}$ is the solid gray curve; the two dotted lines reflect $1 \sigma$ errors in the northsouth intrinsic structure at $43 \mathrm{GHz}$ suggested by Lo et al. (1998). 


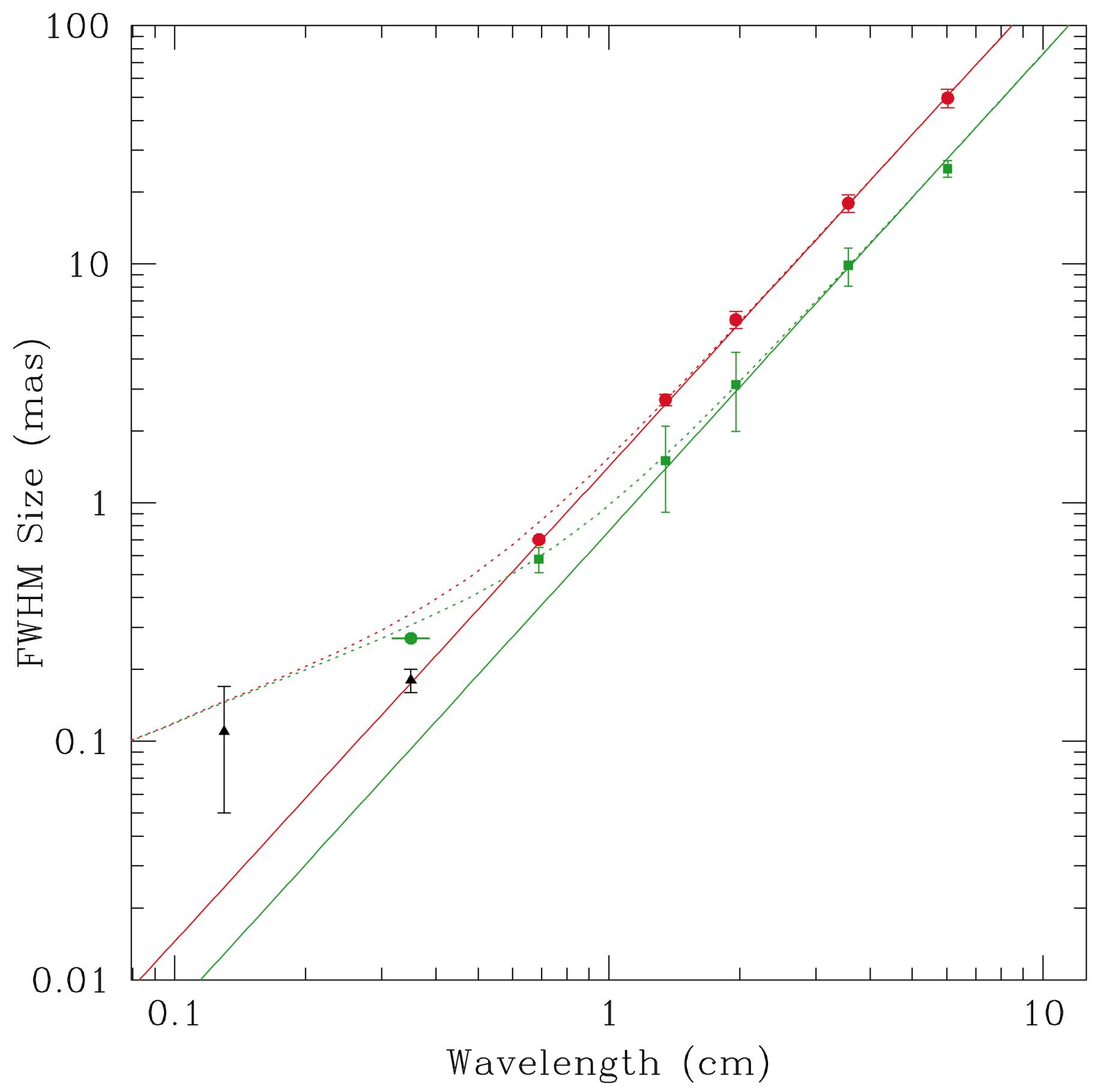

FIG. 7.-Size measurements of Sgr A* from VLBI at multiple wavelengths. Green squares are minor-axis sizes and red circles are major-axis sizes of best-fit elliptical models at wavelengths less than $3.5 \mathrm{~mm}$ from Lo et al. (1998). Green and red solid lines are $\lambda^{2}$ scattering relations given by Lo et al. (1998). The $3.5 \mathrm{~mm}$ upper limit to the size in the north-south direction from this work is shown as a horizontal green line. The north-south observed size predicted from the estimated $7 \mathrm{~mm}$ intrinsic structure (model D in Table 2) is the green filled circle, almost exactly at the upper size limit. Black triangles show the sizes of circular Gaussian models fit to the $3.5 \mathrm{~mm}$ data described in this work and to $1.4 \mathrm{~mm}$ VLBI data (Krichbaum et al. 1998). Hybrid ADAF model sizes from Özel et al. (2000) are added in quadrature to both the minor- and major-axis scattering sizes and are shown as the green and red dotted lines, respectively. The observed sizes predicted by the ADAF model exceed the $3.5 \mathrm{~mm}$ upper limit.

Robinson 1992) is the proper method for determining the statistical significance of a drop in $\chi^{2}$ when additional model parameters are added. The result of such an analysis is based on trying to fit both circular and elliptical Gaussian models to pure noise and calculating the differences in $\chi^{2}$. Given the number of data points in our observations, we find that a $\Delta \chi^{2}=5$ between the two models occurs at least $12 \%$ of the time when fitting pure noise. The better fit of model A, therefore, is only significant at the $1.5 \sigma$ level, and we conclude that the data presented here are adequately fitted by a circular Gaussian. A similar test has not yet been performed for previous $7 \mathrm{~mm}$ wavelength data sets (Lo et al. 1998; Bower \& Backer 1998), and such an analysis would be useful in determining the significance of their results.

Looking for departures from extrapolated scattering sizes due to intrinsic structure is difficult with current arrays. 
Based on contours of $\Delta \chi^{2}=14$, we can limit the elongation in a position angle of $-10^{\circ}$ to less than 0.27 mas. At $7 \mathrm{~mm}$, the effects of intrinsic structure are small, and imaging can be influenced by the calibration of the VLBA antennas. Previously reported measurements at $3.5 \mathrm{~mm}$ by Rogers et al. (1994) and at $1.4 \mathrm{~mm}$ by Krichbaum et al. (1998) have insufficient baselines to be able to rely on closure and are consequently limited by calibration errors. Closure phases derived by summing interferometric phase over station triplets are generally consistent with a zero value. This constrains possible brightness distributions to be symmetric about the origin, a characteristic shared by all the models tested here. Adding secondary components to the Sgr A* structure produces marginally better fits to the closurephase data, but not enough to justify the additional degrees of freedom. The closure-amplitude data presented here show no evidence of asymmetry.

Assuming the scattering and intrinsic structure add in quadrature, limits on the intrinsic size for Sgr A* in position angles of $-10^{\circ}$ and $80^{\circ}$ are 0.25 and 0.13 mas, respectively. For a 1.4 Jy source, these limits correspond to a minimum brightness temperature of $7 \times 10^{9} \mathrm{~K}$, nearly half the value derived in Rogers et al. (1994). The essential difference between the two results is the assumed structure, which Rogers et al. (1994) models as a circular Gaussian. The limits derived here assume an elliptical model, allowing for an extension in the north-south direction and consequent increase in size.

Özel, Psaltis, \& Narayan (2000) have recently computed new ADAF models incorporating generalized electron energy distributions that reproduce the observed Sgr A* emission spectrum. Their size estimate at $7 \mathrm{~mm}$ closely matches the size of the north-south extension seen by Lo et al. (1998), but is much larger than the upper limit to the intrinsic size set in the east-west direction. The spherical ADAF models may have limited applicability to Sgr $\mathrm{A}^{*}$, if an intrinsic ellipticity or asymmetry is verified at shorter wavelengths. At $3.5 \mathrm{~mm}$, the ADAF model (Özel et al. 2000) predicts an intrinsic size of 0.29 mas, well over the $3 \sigma$ upper limit in the north-south direction derived here (Fig. 7). In the AGN model of Falcke \& Biermann (1999), the estimate of intrinsic size for Sgr A* at $7 \mathrm{~mm}$ is used to constrain parameters of the relativistic jet, and the emission region size varies as $\lambda^{0.89 \chi}$, where $\chi$ is a factor related to the jet orientation. An extrapolation to $3.5 \mathrm{~mm}$ gives a size of 0.24 mas, larger than the $3.5 \mathrm{~mm}$ east-west size limit, but consistent with the north-south limits.

\section{SUMMARY}

We have used closure-amplitude information from a 86 $\mathrm{GHz}$ VLBI array to determine a size estimate of Sgr A* without the need to calibrate the array with a priori information. With an assumption of elliptical Gaussian structure, the data are consistent with interstellar scattering of an unresolved source. Our results also do not exclude extrapolations from estimates of intrinsic size at $7 \mathrm{~mm}$ wavelength as long as the intrinsic size evolves faster than $\lambda^{0.9}$. A best-fit elliptical model does exhibit an elongation in the northsouth direction, but the improvement in fit over a circular Gaussian model was found to be of marginal statistical significance. The north-south $u-v$ coverage of the VLBI array is sufficient to constrain an intrinsic extension in the $-10^{\circ}$ position angle to be less than 0.25 mas. Emission models that reproduce the spectrum of Sgr A* predict sizes for Sgr A* that are at or above this size limit.

We thank the staff at the participating observatories for help with the observations and the staff of Haystack Observatory for correlating the data. We also thank Ramesh Narayan and Feryal Özel for making data on their ADAF models available to us prior to publication.
Alberdi, A., et al. 1993, A\&A, 277, L1

Alef, W., \& Porcas, R. W. 1986, A\&A, 168, 365

Backer, D., \& Sramek, R. A. 1999, ApJ, 524, 805

Backer, D. C., Zensus, J. A., Kellerman, K. I., Reid, M., Moran, J. M., \& Lo, K. Y. 1993, Science, 262, 1414

Bevington, P. R., \& Robinson, D. K. 1992, Data Reduction and Error Analysis for the Physical Sciences (2d ed.; New York: McGraw-Hill) Bower, G. C., \& Backer, D. C. 1998, ApJ, 496, L97

Bower, G. C., Falcke, H., Backer, D. C., \& Wright, M. C. H. 1999, in ASP Conf. Ser. 186, The Central Parsecs of the Galaxy, ed. H. Falcke, A. Cotera, W. J. Duschl, F. Melia, \& M. J. Rieke (San Francisco: ASP), 80

Cornwell, T. 1995, in ASP Conf. Ser. 82, Very Long Baseline Interferometry and the VLBA, ed. J. A. Zensus, P. J. Diamond, \& P. J. Napier (San Francisco: ASP), 39

Eckart, A., \& Genzel, R. 1997, MNRAS, 284, 576

Falcke, H., \& Biermann, P. L. 1999, A\&A, 342, 49

Frail, D. A., Diamond, D. J., Cordes, J. M., \& van Langevelde, H. J. 1994, ApJ, 427, L43

Ghez, A. M., Klein, B. L., Morris, M., \& Becklin, E. E. 1998, ApJ, 509, 678

Jauncy, D. L., et al. 1989, AJ, 98, 44

Jennison, R. C. 1958, MNRAS, 118, 276

Krichbaum, T. P., et al. 1998, A\&A, 335, 106L 1993, A\&A, 274, L37

Lo, K. Y., Backer, D. C., Ekers, R. D., Kellermann, K. I., Reid, M., \& Moran, J. M. 1985, Nature, 315, 124

\section{REFERENCES}

Lo, K. Y., Shen, Z.-Q., Zhao, J.-H., \& Ho, P. T. P. 1998, ApJ, 508, L61 .1999, in ASP Conf. Ser. 186, The Central Parsecs of the Galaxy, ed. H. Falcke, A. Cotera, W. J. Duschl, F. Melia, \& M. J. Rieke (San Francisco: ASP), 72

Marcaide, J. M., et al. 1992, A\&A, 258, 295

Melia, F. 1992, ApJ, 387, L25

Narayan, R., Yi, I., \& Mahadevan, R. 1995, Nature, 374, 623

Özel, F., Psaltis, D., \& Narayan, R. 2000, ApJ, 541, 234

Pearson, T. J. 1995, in ASP Conf. Ser. 82, Very Long Baseline Interferometry and the VLBA, ed. J. A. Zensus, P. J. Diamond, \& P. J. Napier (San Francisco: ASP), 267

Quataert, E., Narayan, R., \& Reid, M. J. 1999, ApJ, 517, L101

Readhead, A. C. S., Walker, R. C., Pearson, T. J., \& Cohen, M. H. 1980, Nature, 285,137

Reid, M. J., Readhead, A. C. S., Vermeulen, R. C., \& Treuhaft, R. N. 1999, ApJ, 524, 816

Rogers, A. E. E., Doeleman, S. S., \& Moran, J. M. 1995, AJ, 109, 1391

Rogers, A. E. E., et al. 1994, ApJ, 434, L59 1974, ApJ, 193, 293

Scharf, L. L. 1991, Statistical Signal Processing: Detection, Estimation, and Time Series Analysis (New York: Addison-Wesley)

Schwarz, U. J. 1978, A\&A, 65, 345

Trotter, A. S., Moran, J. M., \& Rodríguez, L. F. 1998, ApJ, 493, 666

Wright, M. C. H., Carlstrom, J. E., Plambeck, R. L., \& Welch, W. J. 1990, AJ, 99,1299

Zylka, P., Mezger, P. G., \& Lesch, H. 1992, A\&A, 261, 119 